Ferrata Storti Foundation

\title{
Predicting sinusoidal obstruction syndrome after allogeneic stem cell transplantation with the EASIX biomarker panel
}

Haematologica 2021

Volume 106(2):446-453

\section{Correspondence:}

OLAF PENACK

olaf.penack@charite.de

Received: September 26, 2019.

Accepted: January 22, 2020.

Pre-published: January 23, 2020.

https://doi.org/10.3324/haematol.2019.238790

(C)2021 Ferrata Storti Foundation

Material published in Haematologica is covered by copyright. All rights are reserved to the Ferrata Storti Foundation. Use of published material is allowed under the following terms and conditions:

https://creativecommons.org/licenses/by-nc/4.0/legalcode. Copies of published material are allowed for personal or internal use. Sharing published material for non-commercial purposes is subject to the following conditions:

https://creativecommons.org/licenses/by-nc/4.0/leǵalcode, sect. 3. Reproducing and sharing published material for commercial purposes is not allowed without permission in writing from the publisher.

\author{
Sihe Jiang,,${ }^{1 *}$ Olaf Penack,,${ }^{1 *}$ Tobias Terzer, ${ }^{2}$ David Schult, ${ }^{3}$ \\ Joshua Majer-Lauterbach, ${ }^{3}$ Aleksandar Radujkovic, ${ }^{3}$ Igor W. Blau, ${ }^{1}$ \\ Lars Bullinger, ${ }^{1}$ Carsten Müller-Tidow, ${ }^{3}$ Peter Dreger ${ }^{3}$ and Thomas Luft ${ }^{3}$
}

${ }^{1}$ Charité Universitätsmedizin Berlin, Campus Virchow Clinic, Hematology, Oncology and Tumorimmunology, Berlin; ${ }^{2}$ Biostatistics, German Cancer Research Centre, Heidelberg and ${ }^{3}$ Medicine V, University Hospital Heidelberg, Heidelberg, Germany

$\star \mathrm{S} J$ and $\mathrm{OP}$ contributed equally as co-first authors.

\section{ABSTRACT}

$\mathrm{N}$ o biomarker panel has been established for prediction of sinusoidal obstruction syndrome/veno-occlusive disease (SOS/VOD), a major complication of allogeneic stem cell transplantation (alloSCT). We compared the potential of the Endothelial Activation and Stress Index (EASIX), based on lactate dehydrogenase, creatinine, and thrombocytes, with that of the SOS/VOD clinical risk score of the Center for International Blood and Marrow Transplant Research (CIBMTR) to predict SOS/VOD in two independent cohorts. In a third cohort, we studied the impact of endothelium-active prophylaxis with pravastatin and ursodeoxycholic acid (UDA) on SOS/VOD risk. The cumulative incidence of SOS/VOD within 28 days after alloSCT in the training cohort (Berlin, 2013-2015, $\mathrm{n}=446$ ) and in the validation cohort (Heidelberg, 2002-2009, $\mathrm{n}=380$ ) was $9.6 \%$ and $8.4 \%$, respectively. In both cohorts, EASIX assessed at the day of alloSCT (EASIX-d0) was significantly associated with SOS/VOD incidence $(P<0.0001)$, overall survival $(O S)$, and non-relapse mortality (NRM). In contrast, the CIBMTR score showed no statistically significant association with SOS/VOD incidence, and did not predict OS and NRM. In patients receiving pravastatin/UDA, the cumulative incidence of SOS/VOD was significantly lower at $1.7 \%$ (Heidelberg, 2010-2015, $\mathrm{n}=359, P<0.0001$ ) than in the two cohorts not receiving pravastatin/UDA. The protective effect was most pronounced in patients with high EASIX-d0. The cumulative SOS/VOD incidence in the highest EASIX-d0 quartiles were $18.1 \%$ and $16.8 \%$ in both cohorts without endothelial prophylaxis as compared to $2.2 \%$ in patients with pravastatin/UDA prophylaxis $(P<0.0001)$. EASIX-d0 is the first validated biomarker for defining a subpopulation of alloSCT recipients at high risk for SOS/VOD. Statin/UDA endothelial prophylaxis could constitute a prophylactic measure for patients at increased SOS/VOD risk.

\section{Introduction}

Sinusoidal obstruction syndrome (SOS), also known as veno-occlusive disease (VOD), is a potentially fatal complication after allogeneic stem cell transplantation (alloSCT). ${ }^{1-3}$ Clinical management of SOS/VOD remains challenging, since there are no standardized predictive tools ${ }^{4}$ and diagnostic criteria are not uniform. ${ }^{2,46}$ The reported incidences of SOS/VOD after alloSCT range from 5.3\% to $13.7 \%$ and vary depending on conditioning regimens, type of transplant, diagnostic criteria, patient characteristics, and other factors. ${ }^{7-9}$

The pathophysiology of SOS/VOD is characterized by endothelial injury caused by the conditioning regimen as well as pre-transplant damage. ${ }^{1,2,10,11}$ The resulting post-sinusoidal portal hypertension leads to the clinical syndrome of SOS/VOD. . $, 5,6,11$ In severe SOS/VOD, which is strongly associated with multi-organ failure, mortality remains high., ${ }^{2,811}$ Early detection or prediction of SOS/VOD could allow identification of patients who could benefit from prophylactic meas- 
ures, $^{3}$ and preliminary data show that pre-emptive treatment with defibrotide might be effective, ${ }^{12}$ making tools to predict SOS/VOD highly desirable.

Recently, a SOS/VOD clinical risk score (age, Karnofsky status, sirolimus use, hepatitis B/C status, conditioning regimen, disease type) has been published by the Center for International Blood and Marrow Transplant Research (CIBMTR). ${ }^{13}$ However, this score has not yet been validated outside of the CIBMTR database. In addition, it has been suggested that serum biomarkers, including microparticles and plasminogen activator inhibitor (PAI)1 , may be useful to predict SOS/VOD, ${ }^{14-18}$ but validation and clinical implementation of these non-routine biomarkers will be difficult due to the lack of standardization.

We had previously developed a standard biomarker panel to assess endothelial dysfunction and activation: the Endothelial Activation and Stress Index (EASIX). EASIX is based on the simple formula "lactate dehydrogenase $(\mathrm{LDH})(\mathrm{U} / \mathrm{L}) *$ creatinine $(\mathrm{mg} / \mathrm{dL}) /$ thrombocytes $\left(10^{9}\right.$ cells per L)" and thus calculated using three of the diagnostic parameters of thrombotic microangiopathy (TMA), ${ }^{19}$ which is another endothelial complication after alloSCT. ${ }^{20}$ EASIX has also been shown to predict mortality of patients with acute graft-versus-host disease (GvHD). ${ }^{19}$

With this background, the aim of the current study was to test the SOS/VOD predictive capacity of EASIX compared with that of the CIBMTR score in two independent cohorts.

\section{Methods}

\section{Study population}

For this retrospective cohort analysis, a training cohort and a validation cohort comprising consecutive adult patients who had undergone alloSCT at two independent institutions were investigated. Patients from the training cohort received alloSCT at the Charité - Campus Virchow Clinic, Berlin, between January 2013 and December 2015. The validation cohort consisted of patients who were allografted at the University of Heidelberg between September 2001 and December 2009. Patients undergoing alloSCT in Heidelberg after January 2010 received pravastatin and ursodeoxycholic acid (UDA) as prophylaxis of endothelial complications after alloSCT. To reduce confounding influences, the validation cohort was restricted to the time period before the introduction of pravastatin and UDA. The study was performed according to the Declaration of Helsinki, with written informed consent obtained by all eligible patients. The study has been approved by the institutional review board of both institutions.

\section{Definitions}

SOS/VOD was defined according to the 2016 European Group for Blood and Marrow Transplantation (EBMT) criteria for SOS/VOD diagnosis in adults. ${ }^{4}$ The disease score we applied for the patients classifies the disease stage of the main diseases: acute leukemia, myelodysplastic syndrome, chronic myeloid leukemia, and non-Hodgkin lymphoma/multiple myeloma. The disease stages are assigned according to the remission status at transplant or the phases of chronic myeloid leukemia. The stages include 'early disease stage' (0), 'intermediate disease stage' (1), and 'late stage disease' (2). ${ }^{21}$

\section{CIBMTR sinusoidal obstruction} syndrome/veno-occlusive disease risk score

The SOS/VOD CIBMTR risk score has been established to assess the risk of developing SOS/VOD after alloSCT. ${ }^{13}$ It incorporates age, hepatitis B/C serology, Karnofsky performance status, use of sirolimus prophylaxis, disease, disease status at the time of transplant, and conditioning regimen. It was developed using the CIBMTR database. ${ }^{13}$ We used the 'VOD Risk Calculator' ${ }^{22}$ and recorded the probability of SOS/VOD development for each patient in the two independent cohorts when possible.

\section{EASIX score}

The EASIX score was calculated using the formula: "LDH (U/L) *creatinine $(\mathrm{mg} / \mathrm{dL}) /$ thrombocytes $\left(10^{9}\right.$ cells per $\left.\mathrm{L}\right)$ " as described previously, assessed at the day of alloSCT (EASIX-d0). ${ }^{19}$

\section{Statistical analysis}

The primary objective was prediction of SOS/VOD occurrence. Primary analysis was performed for the binary endpoint "cumulative incidence of SOS/VOD within 28 days after alloSCT" and the time-to-event endpoint "time to VOD (TTV)" which is defined as time from alloSCT to diagnosis of SOS/VOD. Secondary objectives were the prediction of overall survival $(O S)$ and time to nonrelapse mortality (NRM) measured from the day of alloSCT. TTV and NRM were analyzed using competing event models. The competing event for TTV is "non-SOS/VOD-mortality" defined as time from alloSCT to death without prior SOS/VOD, whereas the competing event for NRM is "time to relapse" defined as time from alloSCT to relapse of disease. Further details on statistical analyses are provided in the Online Supplementary Appendix.

\section{Results}

\section{Patients' characteristics}

The training cohort and the validation cohort consisted of 446 and 380 patients, respectively. The baseline characteristics are presented in Table 1 . The patient cohorts were similar with regards to age, sex, conditioning regimen, and SOS/VOD incidence. However, there were significant differences in the categories donor type (more matched unrelated donors in the training cohort), underlying disease (most frequent disease: acute myeloid leukemia, 51\% and $27 \%$ in the training and validation cohort, respectively), stem cell source (bone marrow used more frequently in the validation cohort), and use of anti-thymocyte globulin (ATG) (more commonly used in the training cohort).

\section{EASIX-d0 and sinusoidal obstruction syndrome/veno-occlusive disease risk}

SOS/VOD was diagnosed in 43 patients $(9.6 \%$, median onset day $[d]+9)$ in the training cohort and in 32 patients $(8.4 \%$, median onset $d+7)$ in the validation cohort. In the training cohort, median EASIX-d0 in patients who later went on to develop SOS/VOD was significantly higher than in patients who did not develop SOS/VOD (40.26; interquartile range [IOR] 14.72-80.38 vs. 16.06; IOR 6.0036.54, $P<0.0001$ ) (Figure 1A). These findings were confirmed in the validation cohort, where median EASIX-d0 in patients who subsequently developed SOS/VOD was also significantly higher than in patients who did not develop SOS/VOD (8.64; IOR 3.38-15.40 vs. 2.28; IOR 0.92-7.48, $P<0.0001$ ) (Figure $1 \mathrm{~B}$ ).

Increasing EASIX-d0 was significantly associated with SOS/VOD incidence in the training cohort in both univariable (OR per $\log _{2}$ increase $1.39,95 \% \mathrm{CI}: 1.18-1.66, P=0.0001$ ) and multivariable analysis with the CIBMTR score as confounder (incorporating information on 6 clinical variables) 
Table 1. Baseline characteristics of the three patient cohorts: Berlin (training), Heidelberg no statins/ursodeoxycholic acid (UDA) (validation), and Heidelberg with statins/UDA.

\begin{tabular}{|c|c|c|c|c|c|c|}
\hline & $\begin{array}{c}\text { Berlin cohort } \\
\text { (training cohort) } \\
(n=446)\end{array}$ & $\begin{array}{c}\text { Heidelberg } \\
\text { no statins/ } \\
\text { UDA cohort } \\
\text { (validation cohort) } \\
\text { (n=380) }\end{array}$ & $\begin{array}{l}\text { Heidelberg } \\
\text { with statins/ } \\
\text { UDA cohort } \\
\text { (n=359) }\end{array}$ & $\begin{array}{c}P \\
\text { Berlin vs. } \\
\text { Heidelberg } \\
\text { no statins/UDA }\end{array}$ & $\begin{array}{c}\text { P } \\
\text { Berlin vs. } \\
\text { Heidelberg } \\
\text { with statins/UDA }\end{array}$ & $\begin{array}{c}P \\
\text { Heidelberg } \\
\text { no statins/UDA } \\
\text { vs. Heidelberg } \\
\text { with statins/UDA }\end{array}$ \\
\hline Date of alloSCT & $01 / 2013-12 / 2015$ & 09/2001-01/2010 & $01 / 2010-12 / 2015$ & & & \\
\hline Age at transplant in years - median (range) & $54(18-75)$ & $50(17-70)$ & $56(19-75)$ & & & \\
\hline Recipient sex (n, \%) & & & & 0.67 & 0.942 & 0.764 \\
\hline Female & $172(39)$ & $153(40)$ & $140(39)$ & & & \\
\hline Male & $274(61)$ & $227(60)$ & $219(61)$ & & & \\
\hline Donor sex $(\mathrm{n}, \%)$ & & & & 0.877 & 0.437 & 0.584 \\
\hline Female & $124(28)$ & $121(32)$ & $122(34)$ & & & \\
\hline Male & $274(61)$ & $259(68)$ & $237(66)$ & & & \\
\hline $\mathrm{NA}$ & $48(11)$ & & & & & \\
\hline Donor (n, \%) & & & & $<0.001$ & 0,034 & $<0.001$ \\
\hline Matched related donor & $88(20)$ & $141(37)$ & $94(26)$ & & & \\
\hline Matched unrelated donor & $263(59)$ & $140(37)$ & $196(55)$ & & & \\
\hline Mismatched related donor & $6(1)$ & $14(4)$ & $11(3)$ & & & \\
\hline Mismatched unrelated donor & $89(20)$ & $85(22)$ & $58(16)$ & & & \\
\hline Disease $(\mathrm{n}, \%)$ & & & & $<0.001$ & $<0.001$ & $<0.001$ \\
\hline AML & $229(51)$ & $101(27)$ & $126(35)$ & & & \\
\hline MDS/MPN & $82(18)$ & $53(14)$ & $69(19)$ & & & \\
\hline ALL & $35(8)$ & $39(10)$ & $20(6)$ & & & \\
\hline Lymphoma & $41(9)$ & $96(25)$ & $100(28)$ & & & \\
\hline MM & $39(9)$ & $75(20)$ & $36(10)$ & & & \\
\hline Other & $20(4)$ & $16(4)$ & $8(2)$ & & & \\
\hline Disease score (n, \%) & & & & 0.012 & $<0.001$ & $<0.001$ \\
\hline 0 & $142(32)$ & $131(34)$ & $122(34)$ & & & \\
\hline 1 & 47 (11) & $65(17)$ & $122(34)$ & & & \\
\hline 2 & $248(56)$ & $184(48)$ & $115(32)$ & & & \\
\hline NA & $9(2)$ & & & & & \\
\hline Stem-cell source (n, \%) & & & & $<0.001$ & $<0.001$ & 0.566 \\
\hline Peripheral blood & $443(99)$ & $351(92)$ & $336(94)$ & & & \\
\hline Bone marrow & $2(1)$ & $29(8)$ & $23(6)$ & & & \\
\hline $\mathrm{NA}$ & $1(0)$ & & & & & \\
\hline Conditioning (n, \%) & & & & 0.999 & 0.055 & 0.069 \\
\hline RIC & $341(76)$ & $291(77)$ & $294(82)$ & & & \\
\hline MAC & $105(24)$ & $89(23)$ & $64(18)$ & & & \\
\hline Use of ATG $(n, \%)$ & & & & $<0.001$ & $<0.001$ & $<0.001$ \\
\hline Yes & $399(89)$ & $193(51)$ & $259(72)$ & & & \\
\hline No & 47 (11) & $187(49)$ & $100(28)$ & & & \\
\hline SOSNOD development (EBMT criteria) (n, \%) & & & & 0.472 & $<0.001$ & $<0.001$ \\
\hline SOSNOD & $43(10)$ & $32(8)$ & $6(2)$ & & & \\
\hline No SOSNOD & $401(90)$ & $348(92)$ & $353(98)$ & & & \\
\hline Onset of SOSNOD (median, range) & $d+9(d+3$ to $d+30)$ & $\mathrm{d}+8(\mathrm{~d} 0$ to $\mathrm{d}+24)$ & $d+10(d+1$ to $d+17)$ & & & \\
\hline \multicolumn{7}{|l|}{ Median CIBMTR score (range, IQR) } \\
\hline SOSNOD & $\mathrm{n}=32$, rest & $\mathrm{n}=29$, rest & $\mathrm{n}=5$, rest & & & \\
\hline & of data NA: 1.51 & of data NA: 1.9 & of data NA: 1.7 & & & \\
\hline No SOSNOD & $\begin{array}{c}(0.56-4.48 ; 0.82-2.37) \\
\mathrm{n}=338, \text { rest }\end{array}$ & $\begin{array}{c}(0.3-8.7 ; 1.4-3.1) \\
\mathrm{n}=333, \text { rest }\end{array}$ & $\begin{array}{c}(0.7-3.7 ; 1.0-2.7) \\
\mathrm{n}=347, \text { rest }\end{array}$ & & & \\
\hline & of data NA: 1.01 & of data NA: 1.2 & of data NA: 1.0 & & & \\
\hline & $(0.32-9.72 ; 0.76-1.80)$ & $(0.4-20.6 ; 0.9-2.1)$ & $(0.3-9.1 ; 0.8-1.7)$ & & & \\
\hline \multicolumn{7}{|l|}{ Median EASIX-d0 (range, IQR) } \\
\hline \multirow[t]{3}{*}{ SOSNOD } & $\mathrm{n}=41$, rest & $\mathrm{n}=32$, rest & $\mathrm{n}=6$ rest & & & \\
\hline & of data NA: 40.26: & of data NA: 8.6 & of data NA: 7.4 & & & \\
\hline & $5.23-865.06 ; 14.72-80.38)$ & $(0.2-41.0 ; 3.4-15.4)$ & $(4.0-17.1 ; 5.8-14.8)$ & & & \\
\hline \multirow[t]{3}{*}{ No SOSNOD } & $\mathrm{n}=376$, rest & $\mathrm{n}=348$, rest & $\mathrm{n}=353$, rest & & & \\
\hline & of data NA: 16.06 & of data NA: 2.3 & of data NA: 7.5 & & & \\
\hline & $(0.38-575 ; 6.00-36.54)$ & $(0.2-99.2 ; 0.9-7.5)$ & $(0.2-195.8 ; 2.1-14.9)$ & & & \\
\hline
\end{tabular}

SOS/VOD: sinusoidal obstruction syndrome/veno-occlusive disease; UDA: ursodeoxycholic acid; alloSCT: allogeneic stem cell transplantation; NA: not available; AML: acute myeloid leukemia; MDS: myelodysplastic syndrome; ALL: acute lymphocytic leukemia; CLL: chronic lymphocytic leukemia; MPN: myeloproliferative neoplasms; CML: chronic myeloid leukemia; MM: multiple myeloma; RIC: reduced intensity conditioning; MAC: myeloablative conditioning; ATG: anti-thymocyte globulin; KPS: Karnofsky Performance Score; IQR: interquartile range; CIBMTR: Center for International Blood and Marrow Transplant Research; EBMT: European Group for Blood and Marrow Transplantation; EASIX-d0: EASIX assessed at the day of alloSCT; n: number; d: day. 
(OR per $\log _{2}$ increase 1.31, 95\%CI:1.08-1.59, $P=0.0067$ ) (Figure 2A). Similarly, EASIX-d0 was strongly associated with the incidence of SOS/VOD in the validation cohort (univariable analysis: OR per $\log _{2}$ increase 1.50, 95\%CI: 1.22-1.88, $P=0.0002$; multivariable analysis: $\mathrm{OR}$ per $\log _{2}$ increase 1.57, 95\%CI: 1.26-2.01, $P=0.0001$ ) (Figure 2B).

Based on these data, we have created the EASIX-d0 SOS/VOD calculator for free public use (http://biostatistics.dkfz.de/EASIX/).

\section{Association of EASIX-d0 with overall survival and non-relapse mortality}

In the training cohort, EASIX-d0 was significantly associated with OS and NRM in univariable analysis (OS: HR per $\log _{2}$ increase $1.20,95 \%$ CI: 1.12-1.29, $P<0.0001$; NRM: cause-specific hazard ratio [CSHR] per $\log _{2}$ increase 1.25, $95 \%$ CI: 1.14-1.38, $P<0.0001$ ) (Figure $3 \mathrm{~A}$ and $\mathrm{C}$ ). Likewise, EASIX-d0 was significantly associated with OS and NRM in the validation cohort in univariable analysis (OS: HR per $\log _{2}$ increase $1.10,95 \%$ CI: $1.02-1.18, P=0.0124$; NRM: CSHR per $\log _{2}$ increase 1.18, 95\%CI: 1.06-1.32, $P=0.0024$ ) (Figure $3 \mathrm{~B}$ and $\mathrm{D}$ ).

\section{Association of the CIBMTR clinical risk score with sinusoidal obstruction syndrome/veno-occlusive disease incidence, overall survival and non-relapse mortality}

In the training cohort, a non-significant trend towards a higher median CIBMTR score was observed in patients who subsequently developed SOS/VOD as compared to patients who did not develop SOS/VOD (1.51; IOR 0.822.37 vs. 1.01; IOR $0.76-1.80, P=0.069)$. In the validation cohort, the median CIBMTR score in patients with SOS/VOD was significantly higher than that in patients without SOS/VOD (1.92; IOR 1.44-3.09 vs. 1.20; IOR $0.89-2.15, P=0.00053)$. On time-to-event analysis, however, the association of the CIBMTR score with SOS/VOD incidence was not statistically significant in the training

\section{A Training cohort}

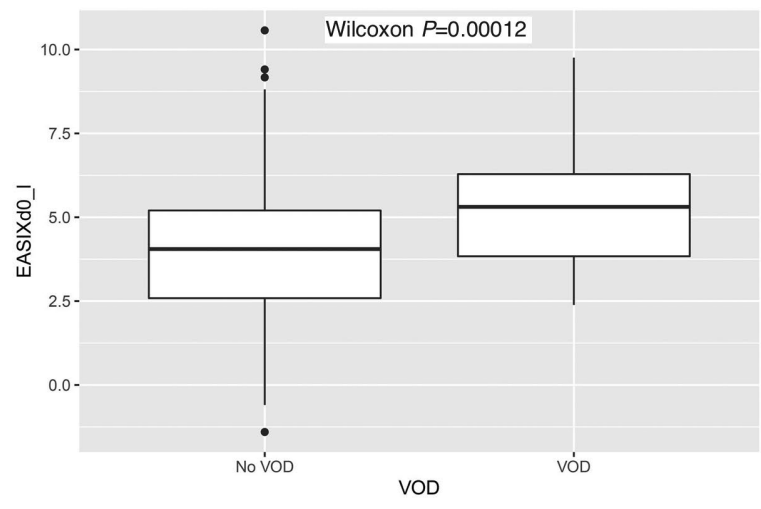

\section{B Validation cohort}

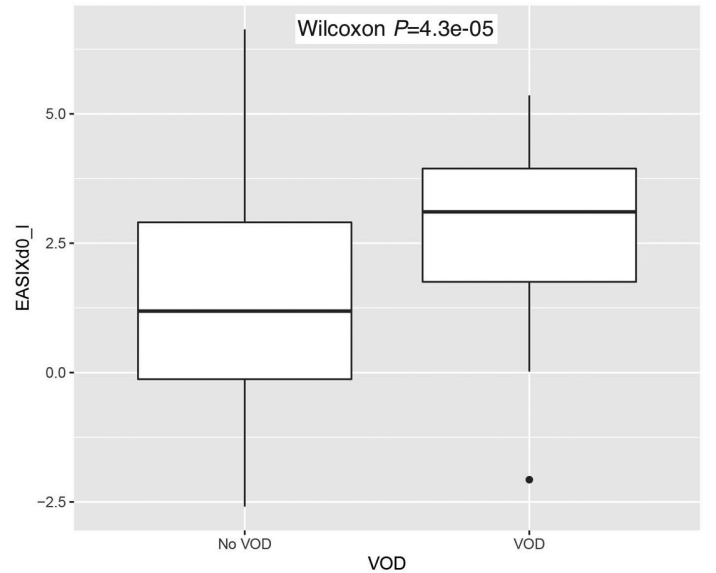

Figure 1. Endothelial Activation and Stress Index (EASIX) assessed at the day of alloSCT (EASIX-d0) in patients without sinusoidal obstruction syndrome/veno-occlusive disease (SOS/VOD) versus EASIX-dO in patients with SOS/VOD. Box plot of EASIX-dO in patients without SOS/VOD (No VOD) versus EASIX-dO in patients with SOS/VOD (VOD)) in (A) the training and (B) the validation cohort.

\section{A Training cohort}

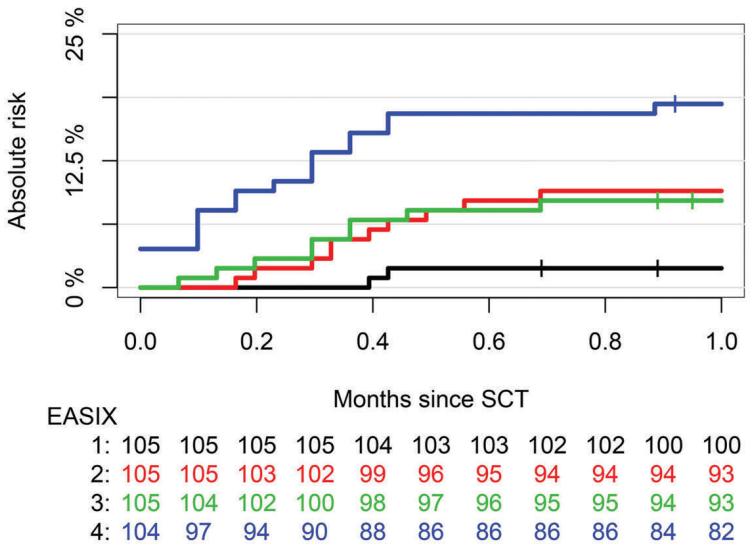

B Validation cohort

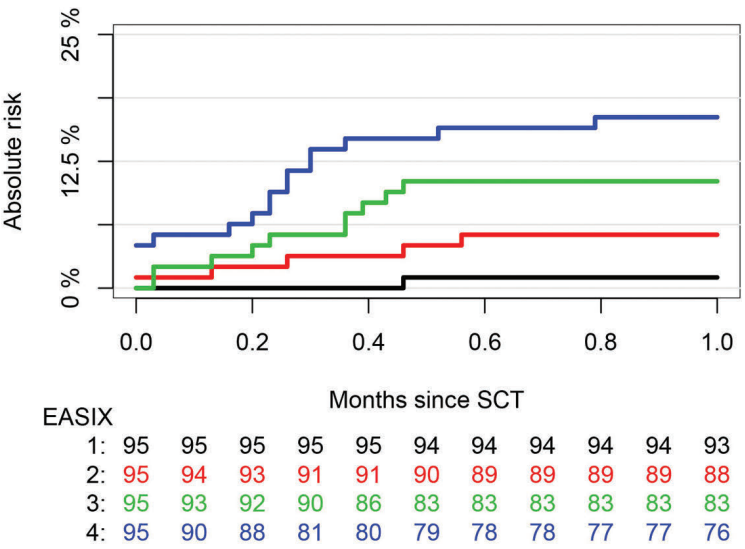

Figure 2. Time to sinusoidal obstruction syndrome/veno-occlusive disease (SOS/VOD) depending on Endothelial Activation and Stress Index (EASIX) assessed at the day of alloSCT (EASIX-d0) quartiles. (A) Training and (B) validation cohort. SCT: stem cell transplantation. 


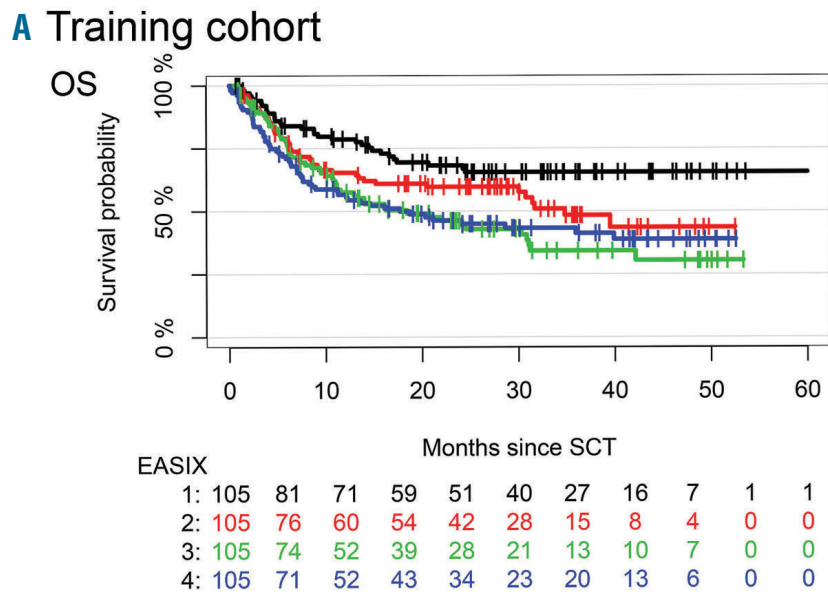

C NRM

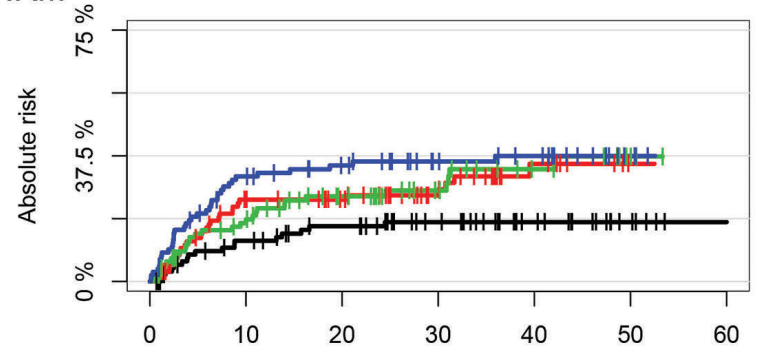

\begin{tabular}{lllllllllll} 
EASIX & \multicolumn{10}{c}{ Months since SCT } \\
1: 105 & 81 & 71 & 59 & 51 & 40 & 27 & 16 & 7 & 1 & 1 \\
2: 105 & 76 & 60 & 54 & 42 & 28 & 15 & 8 & 4 & 0 & 0 \\
3: 104 & 73 & 51 & 39 & 28 & 21 & 13 & 10 & 7 & 0 & 0 \\
4: 105 & 71 & 52 & 43 & 34 & 23 & 20 & 13 & 6 & 0 & 0
\end{tabular}

B Validation cohort

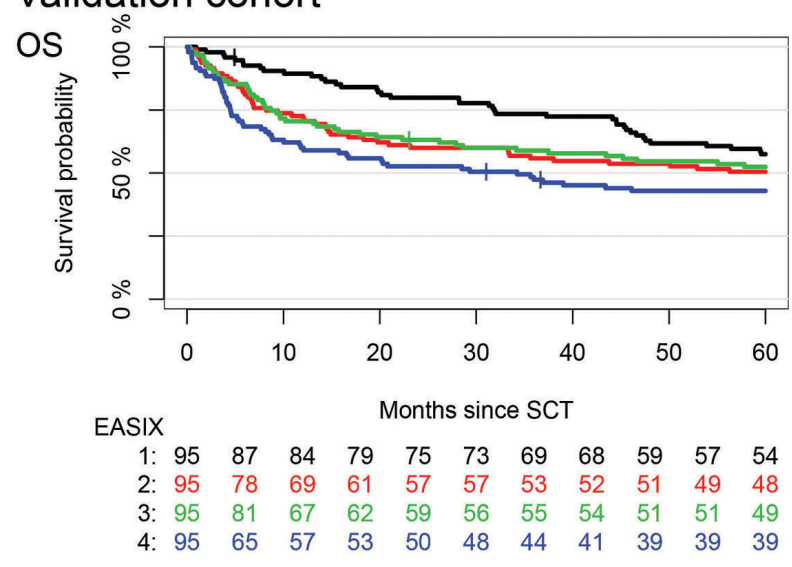

D NRM

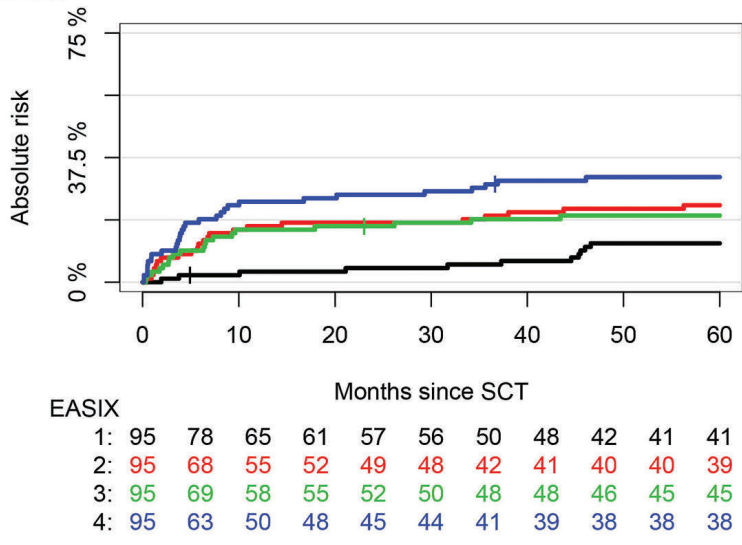

Figure 3. Univariable effect of Endothelial Activation and Stress Index (EASIX) assessed at the day of alloSCT (EASIX-d0) on overall survival (OS) and non-relapse mortality (NRM). Univariable effect of EASIX-dO on OS in (A) the training cohort and (B) the validation cohort. Univariable association of EASIX-dO with NRM in (C) the training cohort and (D) the validation cohort.

cohort (univariable analysis: $\mathrm{OR}$ per $\log _{2}$ increase 1.19 , 95\% CI: 0.87-1.54, $P=0.2288$ ) (Figure 4A) nor in the validation cohort (univariable analysis: $\mathrm{OR}$ per $\log _{2}$ increase 1.07, 95\% CI: 0.92-1.20, $P=0.308$ ) (Figure 4B).

Brier score based on observed SOS/VOD incidence in the training cohort (null model) is 0.0774 in the validation cohort. Inclusion of EASIX-d0 leads to a reduction in the quadratic prediction error (0.0735) of approximately $5 \%$. In contrast, Brier score of the CIBMTR score model (0.0771) is similar to the Brier score of the null model.

In both cohorts, the CIBMTR score was not predictive of OS or NRM (univariable analysis, training cohort, OS: HR per $\log _{2}$ increase 1.08, 95\%CI: $0.95-1.22, P=0.2264$; NRM: CSHR per $\log _{2}$ increase $1.15,95 \% \mathrm{CI}$ : $1.00-1.33$, $P=0.0565$; validation cohort, $\mathrm{OS}$ : $\mathrm{HR}$ per $\log _{2}$ increase 1.03, 95\% CI: 0.97-1.09, $P=0.4126$; NRM: CSHR per $\log _{2}$ increase $0.95,95 \% \mathrm{CI}$ : 0.84-1.08, $\mathrm{P}=0.4648)$.

\section{Effect of pravastatin/ursodeoxycholic acid on sinusoidal obstruction syndrome/veno-occlusive disease incidence}

Patients undergoing alloSCT in Heidelberg after January 2010 received pravastatin and UDA as routine prophylaxis of endothelial complications after alloSCT. In this cohort of 359 consecutive patients transplanted in Heidelberg between January 2010 and December 2015, the SOS/VOD incidence was significantly lower than in the training and validation cohorts treated without endothelial prophylaxis $(1.7 \%$, vs. $9.6 \%$ and $8.4 \%, P<0.0001)$ (Figure $5 \mathrm{~A}$ ). Next, we focused on the effect of pravastatin/UDA prophylaxis on SOS/VOD incidence, NRM and OS in a population at increased risk for SOS/VOD, defined by the highest EASIX-d0 quartile in each of the three cohorts. The patient cohort at increased risk that received pravastatin/UDA showed a significantly lower SOS/VOD incidence (Figure 5B), lower NRM (Figure 5C), and higher OS (Figure 5D) compared to the high-risk patient populations in the training and validation cohorts.

\section{Discussion}

In this retrospective cohort analysis, EASIX-d0 was found to be an independent predictor of SOS/VOD risk, $O S$ and NRM in adult patients receiving alloSCT. EASIX$\mathrm{d} 0$ constitutes the first validated biomarker for defining a subpopulation of alloSCT recipients at high risk for SOS/VOD. It consists of routine laboratory parameters enabling easy implementation in any transplant center. EASIX-d0 seems to be a readily available tool for stratifying patients into high- and low-risk populations, which could be useful to improve clinical management of SOS/VOD, and for identifying patient subsets for clinical trials on SOS/VOD prophylaxis. Outside of clinical stud- 
A Training cohort

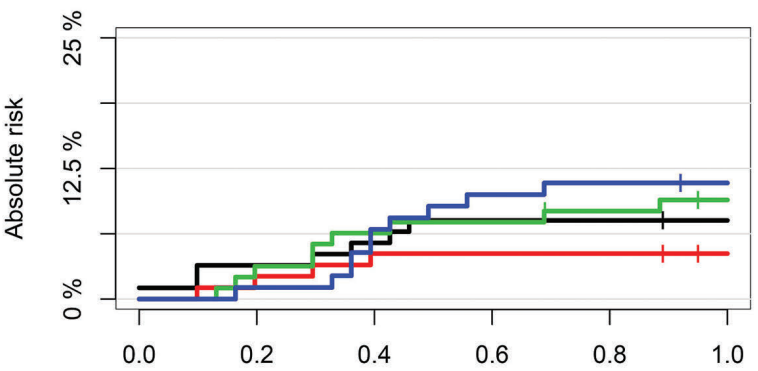

Months since SCT

CIBMTR

\begin{tabular}{|c|c|c|c|c|c|c|c|c|c|}
\hline 1: 93 & 90 & 90 & 89 & 88 & 86 & 86 & 86 & 86 & 84 \\
\hline 2: 92 & 91 & 90 & 89 & 88 & 88 & 88 & 88 & 88 & 86 \\
\hline 3: 95 & 95 & 92 & 90 & 89 & 88 & 88 & 86 & 86 & 85 \\
\hline 90 & 90 & 89 & 89 & 84 & 82 & 81 & 80 & 80 & 80 \\
\hline
\end{tabular}

\section{B Validation cohort}

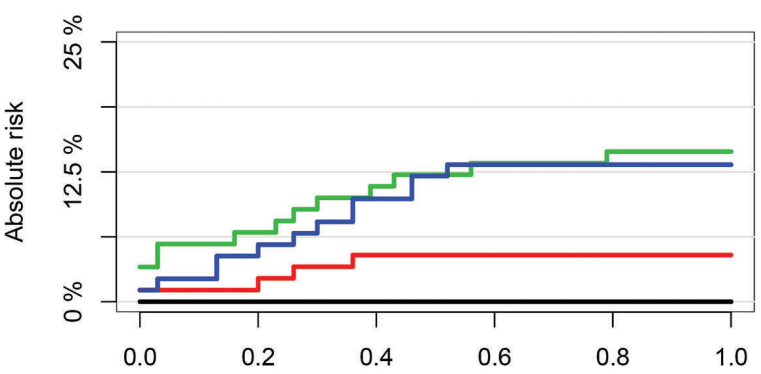

Months since SCT

CIBMTR

1: $\begin{array}{lllllllllll}92 & 92 & 92 & 92 & 92 & 92 & 92 & 92 & 92 & 92 & 92\end{array}$

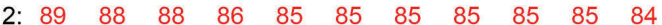

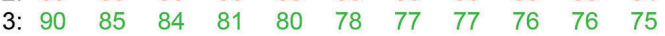

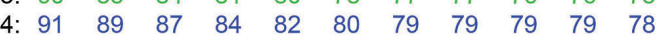

Figure 4. Time to sinusoidal obstruction syndrome/veno-occlusive disease (SOS/VOD) depending on Center for International Blood and Marrow Transplant Research (CIBMTR) score quartiles. (A) Training and (B) validation cohort. SCT: stem cell transplantation.

A Time to SOSIVOD

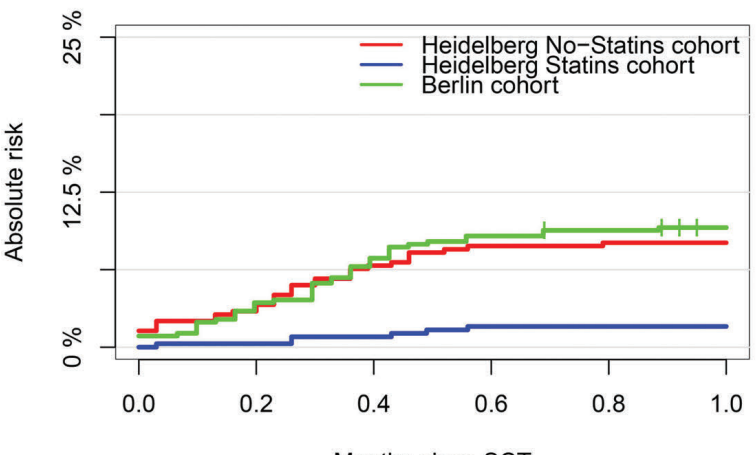

STATINS

Months since SCT

0: $380 \begin{array}{llllllllll}372 & 368 & 357 & 352 & 346 & 344 & 344 & 343 & 343 & 340\end{array}$

1: $\begin{array}{lllllllllll}359 & 358 & 358 & 355 & 353 & 351 & 350 & 350 & 350 & 349 & 348\end{array}$

2: $445 \quad 437 \quad 429422 \quad 413 \quad 406 \quad 403 \quad 400 \quad 400 \quad 395 \quad 390$

C Time to NRM in patients with the highest EASIX-d0 quartile

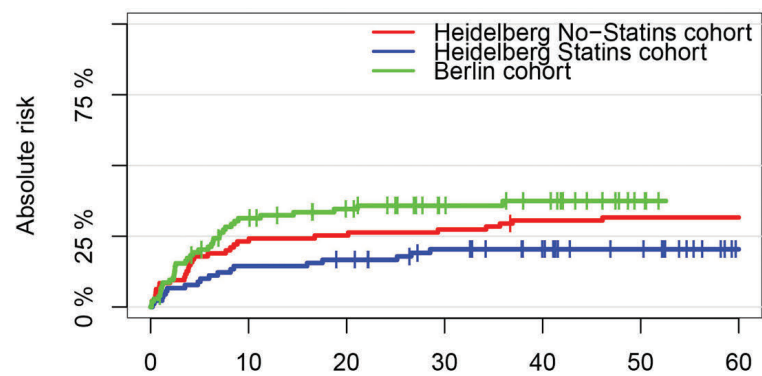

STATINS

Months since SCT

$\begin{array}{lllllllllll}\text { 0: } 95 & 63 & 50 & 48 & 45 & 44 & 41 & 39 & 38 & 38 & 38 \\ \text { 1: } 90 & 62 & 54 & 50 & 44 & 38 & 35 & 27 & 25 & 20 & 12 \\ \text { 2: } 105 & 71 & 52 & 43 & 34 & 23 & 20 & 13 & 6 & 0 & 0\end{array}$

B Time to SOS/VOD in patients with the highest EASIX-d0 quartile

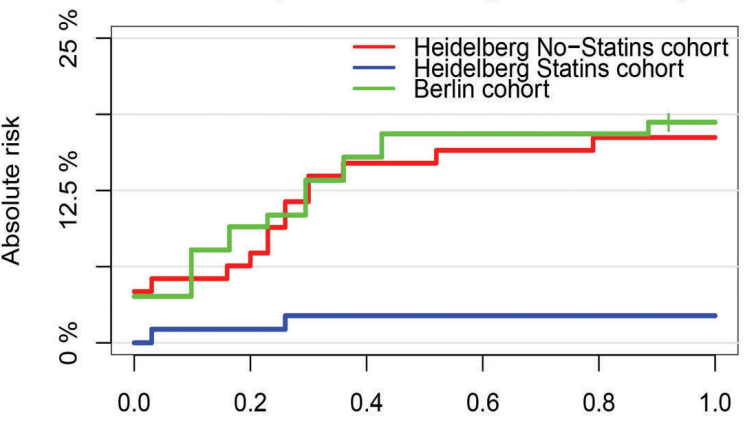

STATINS

Months since SCT

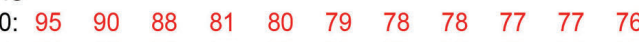

1: $90 \quad 89 \quad \begin{array}{llllllllll}90 & 89 & 87 & 86 & 86 & 86 & 86 & 86 & 86 & 85\end{array}$

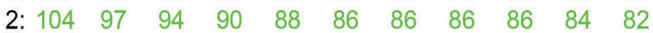

D OS in patients with the highest EASIX-d0 quartile

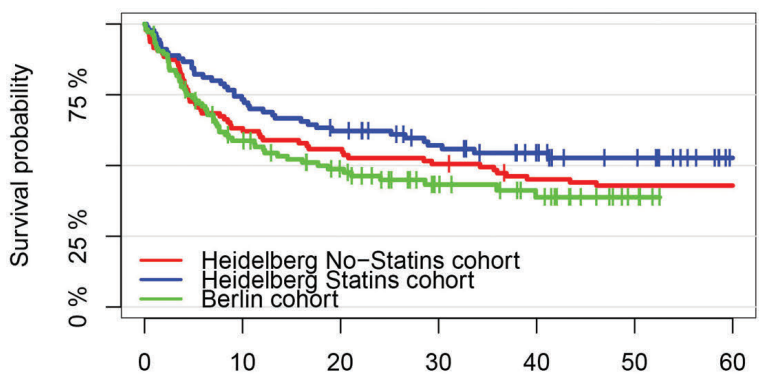

STATINS

Months since SCT

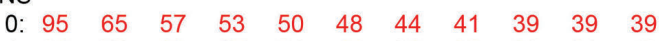

1: $\begin{array}{lllllllllll}90 & 73 & 63 & 57 & 51 & 44 & 38 & 28 & 26 & 21 & 13\end{array}$

2: $\begin{array}{lllllllllll}105 & 71 & 52 & 43 & 34 & 23 & 20 & 13 & 6 & 0 & 0\end{array}$

Figure 5. Effects of pravastatin/ursodeoxycholic acid prophylaxis (blue) compared to the training cohort (green) and the validation cohort (red). (A) Time to sinusoidal obstruction syndrome/veno-occlusive disease (SOS/VOD) in the three cohorts. (B) Time to SOS/VOD in patients with the highest Endothelial Activation and Stress Index (EASIX) assessed at the day of alloSCT (EASIX-dO) quartiles in the three cohorts. (C) Time to non-relapse mortality (NRM) in patients with the highest EASIX-dO quartile in the three cohorts. (D) Overall survival (OS) in patients with the highest EASIX-dO quartile in the three cohorts. SCT: stem cell transplantation. 
ies, patients with high EASIX-d0 scores might benefit from closer monitoring of emerging clinical SOS/VOD signs and early interventions.

EASIX-d0 has to be put into perspective with the CIBMTR SOS/VOD clinical risk score, which has been recently described as a predictive tool to identify patients at high risk of developing SOS/VOD..$^{13}$ The CIBMTR score has been established using a large sample from the CIBMTR database and consists of select baseline parameters which are partly fixed (age, hepatitis B/C serology, Karnofsky performance score, diagnosis, disease status at transplant) and partly subject to intervention (use of sirolimus, conditioning regimen). It has been shown to be predictive for SOS/VOD but not for NRM and OS. ${ }^{13}$ In the present study, the CIBMTR score exhibited only a weak association with SOS/VOD incidence and no association with NRM or OS. One explanation for this discrepancy might be that the two European cohorts investigated in the current study differed in some respects from the CIBMTR cohort. First, in vivo T-cell depletion with ATG was administered in most patients in the two European cohorts, whereas the majority of the CIBMTR patients did not receive ATG for GvHD prophylaxis. ${ }^{13}$ Second, most patients in our two cohorts were conditioned with reduced intensity conditioning (RIC), whereas only a minority of patients from the CIBMTR database received an RIC regimen. ${ }^{13}$ Third, sirolimus was administered in $8 \%$ of the patients from the CIBMTR database, ${ }^{13}$ whereas none of our patients received sirolimus prophylaxis. This is relevant because sirolimus was a risk factor for SOS/VOD development in the CIBMTR analysis. In addition, registry data might be prone to consistency deficits, while we had immediate access to the primary data ensuring the high quality of the data used in our study. Of note, the CIBMTR score has been primarily validated in a large population. We have validated the current EASIX-VOD score in smaller cohorts. Therefore, further validation is needed. The EBMT is currently conducting a prospective non-interventional study on the value of the EASIX score to predict alloSCT-related endothelial complications. Furthermore, we expect that data from several retrospective cohorts in different centers will soon be available.

Based on previous publications on the efficacy of UDA and statins in the protection of the endothelium, ${ }^{23,24}$ the Heidelberg alloSCT group decided to administer pravastatin and UDA as prophylaxis for alloSCT recipients transplanted after January 2010. UDA is a synthetic bile acid that reduces the incidence of SOS/VOD, and is associated with less liver toxicity and better survival rates. ${ }^{3,25}$ Statins have not been extensively studied for the prevention of endothelial complications. However, its pleiotropic effects include, besides the inhibition of cholesterol synthesis, improving endothelial function, reducing oxidative stress and inflammation, and decreasing thrombogenic properties. ${ }^{26,27}$ Statins may therefore be beneficial in the prevention of endothelial complications. Accordingly, we observed a reduction of endothelial post-transplant complications, as previously shown in $\mathrm{TMA}^{20}$ and refractory $\mathrm{GvHD}^{28}$ upon implementation of statin/UDA endothelial prophylaxis.

In the present study, the SOS/VOD incidence was markedly reduced after the introduction of pravastatin/UDA prophylaxis. These protective effects, both in terms of SOS/VOD risk reduction and lower NRM and overall mortality, were particularly pronounced in patients with high EASIX-d0 scores, as compared to highrisk patients who did not receive pravastatin/UDA. This suggests that patients at high risk for SOS/VOD may benefit most from prophylactic SOS/VOD strategies.

Limitations of our study are its retrospective design and the validation in only one independent cohort of patients with similar patients' characteristics, which are typical for adult European alloSCT transplant centers. Therefore, the results cannot be extrapolated to pediatric alloSCT populations. Since EASIX-d0 is very easy to assess, any transplant center now has the opportunity to evaluate its potential in their respective patient population and we hope that more data on different patient populations, including pediatric patients or haploidentical transplantation, will soon be available. To facilitate this process, we have created the EASIX-d0 SOS/VOD calculator for free public use (http://biostatistics.dkfz.de/EASIX/).

In conclusion, EASIX-d0 seems to be an easy-to-use biomarker for identifying patient populations at high risk for SOS/VOD, and thus could be a promising tool both for clinical trials and tailored monitoring strategies. Statin/UDA endothelial prophylaxis could be an option to overcome the increased SOS/NOD risk indicated by high EASIX-d0 scores.

\section{Disclosures}

Olaf Penack has received research support and honorarium as a speaker from Jazz Pharmaceuticals. Thomas Luft has received research support from Jazz Pharmaceuticals and Neovii.

The remaining authors declare no competing interests.

\section{Contributions}

SJ, OP and TL designed the study, performed research, analysed data and wrote the manuscript; DS, AR and JM-L performed research; TT analyzed data; IWB, LB, CM-T and PD designed the study and edited the manuscript. All authors have read the manuscript and have agreed to submit it.

\section{Acknowledgments \\ The authors would like to thank Jazz Pharmaceuticals for financial support.}

\section{Funding}

This study was partly financially supported by Jazz Pharmaceuticals. The company played no role in the acquisition or analyses of data. Jazz was not involved in the writing or editing of the manuscript.

\section{References}

1. Mohty M, Malard F, Abecassis M, et al. Sinusoidal obstruction syndrome/venoocclusive disease: current situation and perspectives - a position statement from the
European Society for Blood and Marrow Transplantation (EBMT). Bone Marrow Transplant. 2015;50(6):781-789.

2. Mcdonald GB, Sharma P, Matthews DE, Shulman HM, Thomas ED. Venocclusive disease of the liver after bone marrow transplantation: diagnosis, incidence, and predis- posing factors. Hepatology. 1984;4(1):116 122.

3. Carreras E. How I manage sinusoidal obstruction syndrome after haematopoietic cell transplantation. Br J Haematol. 2014; 168(4):481-491.

4. Mohty M, Malard F, Abecassis M, et al 
Revised diagnosis and severity criteria for sinusoidal obstruction syndrome/venoocclusive disease in adult patients: a new classification from the European Society for Blood and Marrow Transplantation. Bone Marrow Transplant. 2016;51(7):906-912.

5. Jones RJ, Lee KS, Beschorner WE, et al. Venoocclusive disease of the liver following bone marrow transplantation. Transplantation. 1987:44(6):778-783.

6. McDonald GB, Hinds MS, Fisher LD, et al. Veno-occlusive disease of the liver and multiorgan failure after bone marrow transplantation: a cohort study of 355 patients. Ann Intern Med. 1993;118(4):255-267.

7. Carreras E, Bertz H, Arcese W, et al. Incidence and outcome of hepatic venoocclusive disease after blood or marrow transplantation: a prospective cohort study of the European Group for Blood and Marrow Transplantation. Blood. 1998;92 (10):3599-3604

8. Coppell JA, Richardson PG, Soiffer R, et al. Hepatic veno-occlusive disease following stem cell transplantation: incidence, clinical course, and outcome. Biol Blood Marrow Transplant. 2010;16(2):157-168.

9. Dalle J-H, Giralt SA. Hepatic veno-occlusive disease after hematopoietic stem cell transplantation: risk factors and stratification, prophylaxis, and treatment. Biol Blood Marrow Transplant. 2016;22(3):400-409.

10. Carreras E, Diaz-Ricart M. The role of the endothelium in the short-term complications of hematopoietic SCT. Bone Marrow Transplant. 2011;46(12):1495-1502.

11. Bearman S. The syndrome of hepatic venoocclusive disease after marrow transplantation. Blood. 1995;85(11):3005-3020.

12. Richardson PG, Smith AR, Triplett BM, et al. Earlier defibrotide initiation post-diagnosis of veno-occlusive disease/sinusoidal obstruction syndrome improves day +100 survival following haematopoietic stem cell transplantation. Br J Haematol. 2017; 178(1):112-118

13. Strouse C, Zhang Y, Zhang M-I, et al. Risk score for the development of veno-occlusive disease after allogeneic hematopoietic cell transplant. Biol Blood Marrow Transplant. 2018;24(10):2072-2080

14. Salat C, Holler E, Kolb H-J, et al. Plasminogen activator inhibitor-1 confirms the diagnosis of hepatic veno-occlusive disease in patients with hyperbilirubinemia after bone marrow transplantation. Blood. 1997;89(6):2184-2188.

15. Tanikawa S, Mori S, Ohhashi K, et al. Predictive markers for hepatic veno-occlusive disease after hematopoietic stem cell transplantation in adults: a prospective single center study. Bone Marrow Transplant. 2000;26(8):881-886.

16. Cutler C, Kim HT, Ayanian S, et al. Prediction of veno-occlusive disease using biomarkers of endothelial injury. Biol Blood Marrow Transplant. 2010;16(8):1180-1185.

17. Akil A, Zhang O, Mumaw CL, et al. Biomarkers for diagnosis and prognosis of sinusoidal obstruction syndrome after hematopoietic cell transplantation. Biol Blood Marrow Transplant. 2015; 21(10):1739-1745

18. Piccin A, Sartori MT, Bisogno G, et al. New insights into sinusoidal obstruction syndrome: microparticles and VOD. Intern Med J. 2017;47(10):1173-1183

19. Luft $\mathrm{T}$, Benner A, Jodele $\mathrm{S}$, et al. EASIX in patients with acute graft-versus-host disease: a retrospective cohort analysis. Lancet Haematol. 2017;4(9):e414-e423.

20. Zeisbrich M, Becker N, Benner A, et al. Transplant-associated thrombotic microangiopathy is an endothelial complication associated with refractoriness of acute GvHD. Bone Marrow Transplant.
2017;52(10):1399-1405

21. Gratwohl A, Stern M, Brand R, et al. Risk score for outcome after allogeneic hematopoietic stem cell transplantation: a retrospective analysis. Cancer. 2009, 115(20):4715-4726.

22. Center for International Blood \& Marrow Transplant Research. VOD Risk Calculator. 2018. https:// www.cibmtr.org/ Reference Center/Statistical/Tools/Pages/VOD.aspx (Last accessed August 24, 2018).

23. Ruutu T, Eriksson B, Remes K, et al Ursodeoxycholic acid for the prevention of hepatic complications in allogeneic stem cell transplantation. Blood. 2002;100(6):1977 1983

24. Ruutu $\mathrm{T}$, Juvonen $\mathrm{E}$, Remberger $\mathrm{M}$, et al Improved survival with ursodeoxycholic acid prophylaxis in allogeneic stem cell transplantation: long-term follow-up of a randomized study. Biol Blood Marrow Transplant. 2014;20(1):135-138.

25. Cheuk DKL, Chiang AKS, Ha SY, Chan GCF. Interventions for prophylaxis of hepat ic veno-occlusive disease in people undergoing haematopoietic stem cell transplantation. Cochrane Database Syst Rev. 2015;(5):CD009311.

26. Lahera V, Goicoechea M, de Vinuesa SG, et al. Endothelial dysfunction, oxidative stress and inflammation in atherosclerosis: beneficial effects of statins. Curr Med Chem. 2007;14(2):243-248

27. Blum A, Shamburek R. The pleiotropic effects of statins on endothelial function, vascular inflammation, immunomodulation and thrombogenesis. Atherosclerosis. 2009, 203(2):325-330

28. Dietrich S, Okun JG, Schmidt K, et al. High pre-transplant serum nitrate levels predic risk of acute steroid-refractory graft-versushost disease in the absence of statin therapy. Haematologica. 2014;99(3):541-547. 